\title{
Qualitative methods in research on healthcare quality
}

\author{
C Pope, P van Royen, R Baker
}

Qual Saf Health Care 2002;11:148-152

There are no easy solutions to the problem of improving the quality of care. Research has shown how difficult it can be, but has failed to provide reliable and effective ways to change services and professional performance for the better. Much depends on the perspectives of users and the attitudes and behaviours of professionals in the context of their organisations and healthcare teams. Qualitative research offers a variety of methods for identifying what really matters to patients and carers, detecting obstacles to changing performance, and explaining why improvement does or does not occur. The use of such methods in future studies could lead to a better understanding of how to improve quality.

See end of article for authors' affiliations

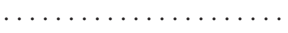

Correspondence to: Dr C Pope, Lecturer in Medical Sociology, Department of Social Medicine, University of Bristol, Bristol BS8 2PR UK; c.pope@bristol.ac.uk
"I went to see Roy Griffiths (architect of the 1984 NHS reforms and supermarket chief executive) in his office at Sainsbury's and while I was talking to him his secretary handed him a piece of paper. He looked at it and said: 'OK'. I asked him: 'What do you mean OK?' and he said: 'My organisation is OK today'. It turned out he had just six measures on that piece of paper and from those he could tell what the state of Sainsbury's health had been the day before; things like the amount of money taken yesterday, the freshness quotient - the amount of stuff on the shelves - the proportion of staff on duty and so on." NHS regional manager quoted in Strong and Robinson.

The above quote illustrates a view of management in the retail sector that was seen in the UK in the 1980s as a role model for health services. The quality of health care could be assured if we could only develop good quantitative measures such as performance indicators, and thus identify problems, make changes, and improve health services. This vision has been tempered with the realisation that the issue of "quality" is more complicated and nebulous than this model of management implies, especially in the case of complex health systems and services. The assessment of quality of services can no longer be confined to simply monitoring such aspects as waiting time, but requires an understanding of the experience of waiting for care-for example, the nature of the clinical environment, the adequacy of communication by and with health professionals, the context and manner in which treatment is delivered, and whether services and care meet expectations. Moreover, it is increasingly recognised that views of quality depend on one's perspective: patients, providers, politicians, and the public may all have contested views of what constitutes high or poor quality care.

The concept of quality in health care is multidimensional and complex and some of the ques- tions we want to ask about the quality of care or services may not be amenable to quantitative measurement. Qualitative research has come to the fore in health and social research by providing ways of answering these sorts of questions, ${ }^{23}$ both in the form of "stand alone" or independent research projects and as a complement to quantitative studies.

The use of qualitative methods in qualitative research involves the systematic collection, organisation, and analysis of textual material derived from talk or observation. It is rooted in the interpretive perspectives found in the humanities and social sciences that emphasise the importance of understanding, from the viewpoint of the people involved, how individuals and groups interpret, experience, and make sense of social phenomena. It is not possible here to elaborate on the origins and theoretical underpinnings of this distinctive approach to research, but it is important to be aware that qualitative research is informed by a quite different paradigm to that which governs quantitative clinical and biomedical research. The emphasis in qualitative research on understanding meanings and experiences makes it particularly useful for quality assessment and for unpacking some of the complex issues inherent to quality improvement. This paper explores some of the qualitative methods that can help to gather information about the delivery of good quality health care and explain variations in health care provision.

\section{THE METHODS}

Qualitative researchers study phenomena and events in their natural settings, often interpreting them in terms of the subjective meanings attached by the individual. Qualitative methods for collecting data include interviews, observation, and analysis of documents. Different methods may be appropriate to different situations and different research questions. In some cases a single method may be used while in others a combination of methods may be employed. In this paper we focus on interview based and observational methods as these are the most commonly used in quality assessment.

\section{Interview based methods}

Individual face to face interviews may be either semi-structured or in-depth. Semi-structured interviews are typically based on a flexible topic guide that provides a loose structure of open ended questions to explore experiences and attitudes. In-depth interviews provide an opportunity to obtain more detail about an issue or experience, and are especially useful for exploring experiences of care. Because this method elicits people's own views and accounts, it can have the 
additional benefit of uncovering issues or concerns that had not been anticipated or considered by the researchers. In order to ensure that really detailed information is gathered, interview methods require experienced researchers with the necessary sensitivity and ability to establish rapport with respondents, to use topic guides flexibly and follow up questions and responses.

Focus groups are similar in structure to face to face interviews but they use the interaction of a group of, typically, 6-8 people to generate data. This allows group members to talk to one another, argue and ask questions, and is especially useful for finding out about shared experiences. Focus groups have been successfully used with users and staff. One adaptation of this method is the "exploration group" in which different healthcare providers who have direct contact with a particular health problem review and discuss some material such as audio or video taped cases or interviews in order to develop an interpretative explanation. ${ }^{4}$

Another variant is the "quality circle". This convenes a small group of healthcare providers and patients who meet at regular times for a determined period to formulate hypotheses or action points to improve quality in health care. ${ }^{5}$

\section{Observation based methods}

The systematic observation of organisational settings, team behaviour, and interactions is especially useful in studying quality issues as it allows researchers to uncover everyday behaviour rather than only relying on interview accounts. These methods are increasingly used in the study of organisation and delivery of care $^{6}$ and can be especially useful in uncovering what really happens in particular healthcare settings-for example, in the study of everyday work in labour wards ${ }^{7}$ - and for formative evaluation of new services.

\section{Narrative based medicine}

Narrative based medicine is one of several patient centred approaches that can give the physician access to the lived experience of their patients. This is the context in which the physician interprets symptoms and signs and in which personal healthcare decisions are made. It can therefore be an approach to understanding how healthcare decisions are made.

\section{SAMPLING}

Qualitative methods are designed to yield detailed and holistic views of the phenomena under study. The aim of qualitative research is not therefore to identify a statistically representative set of respondents or to produce numerical predictions. Qualitative research questions tend to be exploratory and not tied to formal hypothesis testing, so the sampling strategies used in qualitative research are purposive or theoretical rather than representative or probability based. ${ }^{8}$ This means that respondents are sampled based on specific predetermined criteria in order to cover a range of constituencies-for example, different age, social class, and cultural backgrounds (see box 1). ${ }^{9}$ To locate hard to reach individuals or groups, researchers can use "convenience" venues, informants, or social networks.

The sample sizes for interview studies tend to be much smaller than those used in survey or more quantitative research; they may include 30-50 respondents, although this can vary with the research question asked. Similarly, observational studies may be based on a single case study, perhaps focusing on one organisational setting such as a clinic or ward.

\section{ANALYSIS}

Qualitative analyses attempt to preserve the textual form of the data gathered and to generate analytical categories and explanations. This may be done inductively-that is, obtained gradually from the data-or deductively- that is, with a theoretical framework as background (box 2), ${ }^{10}$ either at the
Box 1 Focus group study to obtain information

directly from adolescent young women on their knowledge and expectations concerning contraceptive use and their attitude to healthcare

To obtain a range of views, a purposive direct sampling strategy was followed to organise the focus groups. Four secondary schools with different educational levels were selected because of the correlation with sexual behaviour of adolescents. In each school all 17 year old young women of one small 5th grade class were asked to participate. Each group comprised six or seven participants, with a total of 26. Differences in sexual experiences and social classes fostered lively interaction within the groups. The discussions were tape recorded, transcribed, and analysed by content analysis. Knowledge of the daily use and side effects of contraceptives was insufficient. The general practitioner was the most frequently consulted healthcare provider for the first pill prescription, but for a gynaecological examination they thought they had to visit a gynaecologist. Mothers and the peer group were important in teenagers' decision making and should be considered when communicating with adolescent young women.

Box 2 Investigation of barriers to implementing guidelines for the management of depression in general practice ${ }^{10}$

Information about how general practitioners (34 in the intervention group) managed patients with depression were obtained from review of records and assessment of outcome with a standard patient completed questionnaire. The guidelines were issued to the general practitioners and they were then interviewed individually to identify their personal barriers to acting on the recommendations. The interviews were semi-structured and were recorded and transcribed. Psychological theories of behaviour change were used as the framework for analysing the interviews. The transcripts were repeatedly studied independently by several researchers. An example of the barriers identified through use of a theoretical framework relates to the theory of self-efficacy. Some general practitioners did not feel able to ask about suicide risk because they lacked confidence in their ability to use an appropriate form of words. After the general practitioners had been given suggestions for phrases to use, the proportion of patients whose suicide risk had been assessed increased.

beginning or part way through the analysis as a way of approaching the data.

There are various software packages designed to assist with the organisation and retrieval of qualitative data. Among those most commonly used are QSR NUD* ${ }^{*} T^{11}$ and Atlas Ti. ${ }^{12}$ Some of these packages enable sophisticated analysis, allowing the researcher to make theoretical links within the data set; others identify co-occurring codes and provide opportunities to annotate codes or portions of text. All of these processes are integral to qualitative data analysis, but whether software is used or not, the key point about the analysis is that it relies on systematic and rigorous searching of text for categories and themes. These categories and themes are collected together, compared, and re-analysed to develop hypotheses or theoretical explanations. When conducting this coding analysis the researcher gives consideration to the actual words used, the context, the internal consistency, the specificity of responses that is more based on own experiences 


\section{Box 3 The coding process}

(1) As the researcher comes across an idea or phenomenon a label is attached. A fragment from adolescent girls' focus groups": "I always talk to my mother because I can tell her everything. You can always get reliable information from your mother." Considering the actual words used, possible labels for this fragment are: "communication", "mother", "reliable", "information".

(2) When the idea or phenomenon reappears in the text, the label is once again attached. "If it is really necessary, I will talk to my mother. But I don't like that, but .."

(3) After reading again all codes and fragments, a better formulation of the code can be found; for this fragment, the label "information sources/mother" can be used.

(4) Specificity: responses based on specific experiences are more important. "I always talk to my mother ..." is more specific than "Girls of my class are used to talk to their mother." (5) Codebooks consist of a set of codes that capture the key analytical constructs. Step by step you progress in the level of analysing: raw data $\rightarrow$ description $\rightarrow$ interpretation $\rightarrow$ recommendation.

of respondents, and the big ideas beneath all detailed information. ${ }^{13}$ It is important in this process not to lose sight of the narrative and textual structure of qualitative data, and to pay attention to the context of items of data (box 3). While software packages can assist with this labour intensive process and offer great potential for managing large data sets, they are not a substitute for thorough knowledge or "immersion" in the data which enables the researcher to identify connections and patterns, to make systematic comparisons, and to develop interpretations.

\section{RELIABILITY AND VALIDITY}

When it comes to judging the quality of qualitative research, qualitative methods are often seen as scoring highly in terms of internal validity. By documenting how people really behave in "natural" everyday situations and examining in detail what people mean when they describe their experiences, feelings, attitudes and behaviour, these methods are seen as providing an accurate representation of the phenomena studied. Reliability, which is a particular strength of quantitative research, cannot always be judged so easily within a qualitative study. The settings and groups studied within qualitative research may be unique to the particular context or time period, and it is unlikely that a study can be replicated in the way that a controlled experiment can. Sometimes it is possible to involve other researchers in the analytical process to code the data independently or to discuss emerging themes and categories to try to reach consensus about the interpretation of the data. It is important that a clear account of the data collection and analysis is provided to allow readers to judge the evidence and interpretations presented. This clear exposition is also essential for judging the transferability of findings to other settings or groups.

\section{SOME EXAMPLES OF QUALITATIVE RESEARCH ABOUT THE QUALITY OF HEALTH CARE}

Qualitative research has been used in a number of ways to look at the quality of health care. To illustrate how these methods can inform quality improvement, we focus on three areas where qualitative methods have made a contribution: (1) in identifying salient features of care to inform service delivery and organisation; (2) in exploring organisational and other obstacles to change, notably within the context of healthcare evaluation; and (3) by complementing other research approaches either in the preliminary development of measures or in explaining or implementing findings.

\section{Identifying what really matters to patients and care} providers

Interviews or focus group methods are especially helpful in assessing user views of services and healthcare provision and in revealing why some care is perceived as poor quality. One interview study looked at patients' perceptions of the reassurance provided by rheumatologists and found that the typical methods of imparting reassurance, often by minimising or downplaying the seriousness of the arthritic condition, were frequently misinterpreted by patients. This study showed that clinicians needed to be more aware of patients' own views and experience of health problems and to adapt their explanations and information giving to increase its salience for this group of patients. ${ }^{14}$ Similarly, an Australian study ${ }^{15}$ found that the quality of information and reassurance given to women receiving abnormal cervical smear test results was poor and recommended different ways of organising the service to meet women's information needs and to improve the quality of care.

Qualitative work can be helpful in identifying cultural and social factors that hinder or encourage service use. This information can be directly fed back to healthcare providers to help them improve service delivery (box 1). ${ }^{9}$ In the UK, focus groups with women from ethnic minorities ${ }^{16}$ identified administrative and language barriers which prevented these women from using cervical screening services.

\section{Identifying obstacles to change}

By establishing the reasons behind certain behaviours, qualitative research can help to identify barriers to practice change. Success will be more likely if the methods used to implement change are chosen to address the prevailing barriers. ${ }^{17}$ Interview studies have been used to identify modifiable factors associated with prescribing by general practitioners ${ }^{18}$ and to distinguish doctor and patient related factors that explain a high level of prescribing of antibiotics. ${ }^{19}$ Sometimes qualitative research is helpful in understanding how organisations and teams within them work on a day to day basis. Observational research by Hughes and Griffith $s^{20}$ on rationing in cardiac care conferences and neurorehabilitation meetings showed how decisions differed between these two types of service. Making these decision rules explicit makes it possible to see how this process might be improved or adapted. Elsewhere, interviews with general practitioners were used in a randomised trial of the implementation of guidelines for the management of depression in general practice to tailor intervention strategies to the needs of practitioners. ${ }^{10}$ The identified barriers to change included doctors' perceived ability to assess suicide risk and inform patients about taking their medication (box 2). Further research is needed to elucidate the most efficient methods for identifying barriers to change and to investigate theoretical frameworks that can be used to understand barriers.

The complex structures and behaviours of healthcare organisations are increasingly recognised as critical factors in determining the quality of care. ${ }^{21}$ Qualitative methods offer a potential approach to assisting leaders of organisations to appreciate some of the local issues to be considered when introducing new ideas or transforming systems of care. However, more research is needed to investigate which qualitative methods could be most useful, and in what circumstances they should be used.

Another strength of qualitative research lies in its role within formative evaluation. Qualitative methods can provide insights to the process of policy implementation, identifying where and why this is successful, uncovering initial "teething problems", and suggesting solutions. Qualitative methods have also been used to guide the design of a new "one stop" clinic for women with menstrual problems and to evaluate the service from the patient's perspective. ${ }^{22}$ 
Box 4 Development of a measure of patients' views of care across the primary/secondary interface ${ }^{23}$

The aim was to develop a standard quantitative measure of the views of patients referred from primary to secondary care. In order to identify the issues of concern to patients, a purposeful sample of patients who had been referred to secondary care was identified. Six focus group meetings were held and five patients who could not travel to a meeting were interviewed individually. Two researchers independently studied the transcripts and developed coding schemes. Differences were resolved through discussion. Five main themes emerged from the data:

- getting into care;

- fitting in with staff and systems of care

- knowing what's going on (obtaining information);

- continuity of care;

- limbo (progress through the healthcare system).

Failures in the first four themes led patients to report feeling as though they were not making progress and had been left in a state of limbo.

\section{Complementing other research}

Qualitative methods have long been used to inform more quantitative research approaches, notably assisting with research design and the development of outcome measures. They have been used in preliminary work for surveys to develop and test questionnaires-for example, the development of quantitative measures of patients' views should begin with an exploration of the views of samples of patients using qualitative methods (box 4). ${ }^{23}$

They can also be used as part of the process of dissemination of research evidence, and may be especially helpful in making findings relevant to patients and care providers. Thus, although it can be difficult to incorporate the views of patients or carers, qualitative methods may sometimes be useful in informing recommendations and guidelines. ${ }^{24} 25$ The choice of method will depend on the topic and the evidence base, but patient or carer focus groups or interviews can be appropriate if the guideline is concerned with the interpersonal aspects of care, with very small subgroups of patients or carers, or if the available evidence is limited.

\section{CONCLUSIONS}

This paper has introduced some of the methods of qualitative research and outlined some ways in which they can contribute to research into quality improvement and management of change. Quality improvement is a major goal of the healthcare systems of most developed countries yet, despite almost two decades of research, effective approaches remain elusive. In order to understand better the human and organisational factors that influence the quality and safety of care, researchers

\section{Key messages}

- There are several methods for collecting data in qualitative research, including both interview based methods and observation based methods.

- Sampling methods are theoretical or purposive, and analysis may be inductive or deductive.

- These methods may be used to identify what really matters to patients and carers, and can also be used to explain the obstacles to improvement and why improvement does or does not occur.

- Qualitative methods could make an important contribution to understanding how to improve the quality of health care. should remember the potential role of qualitative methods. Qualitative research encompasses a range of methods that have successfully been used to explore issues of healthcare delivery from patient and provider perspectives. They can help both to illuminate different facets of "quality" and to inform quantitative approaches to researching health care.

\section{BIBLIOGRAPHY}

Suggested introductory books to qualitative research methods:

Denzin NK, Lincoln YS, eds. Handbook of qualitative research. Thousand Oaks: Sage, 1998.

Gantley M, Harding G, Kumar S, Tissier J. An introduction to qualitative methods for health professionals. Master Classes in Primary Care Research No 1 (editors Carter Y, Shaw S, Thomas C). London: Royal College of General Practitioners, 1999.

\section{Authors' affiliations}

C Pope, Lecturer in Medical Sociology, Department of Social Medicine, University of Bristol, Bristol BS8 2PR, UK

P van Royen, Professor of General Practice, Department of General Practice, University of Antwerp, Universiteitsplein 1, 2610 Antwerp, Belgium

R Baker, Professor of Quality in Health Care, Clinical Governance

Research \& Development Unit, Department of General Practice \& Primary Health Care, University of Leicester, Leicester LE5 4PW, UK

\section{REFERENCES}

1 Strong P, Robinson J. The NHS: under new management. Milton Keynes: Open University Press, 1990: 81.

2 Mays N, Pope C. Reaching the parts other methods cannot reach: an introduction to qualitative methods in health and health services research. BM 1995;311:42-5.

3 Malterud K. Qualitative research: standards, challenges, and guidelines. Lancet $2001 ; 358: 483-8$.

4 Wijnen G, Schillemans L, Hermann I. Introduction of the WVVH prevention card in general practice (in Dutch). Huisarts $\mathrm{Nu}$ 1993;22:17-21

5 Schillemans L, De Grande L, Remmen R. Using quality circles to evaluate the efficacy of primary health care. In: Conner RF, Hendricks $M$, eds. International innovations in evaluation methodology. New directions in program evaluation 42. San Francisco: Jossey-Bass, 1989: 19-27.

6 Murphy E. Micro-level qualitative research. In: Fulop N, Allen P, Clarke $A$, et al, eds. Studying the organisation and delivery of health services research methods. London: Routledge, 2001: 40-55.

7 Hunt S, Symonds A. The social meaning of midwifery. Basingstoke: Macmillan, 1995

8 Patton M. Qualitative evaluation and research methods. Newbury Park, CA: Sage, 1990.

9 Peremans L, Hermann I, Avonts D, et al. Contraceptive knowledge and expectations by adolescents: an explanation by focus groups. Patient Educat Counsel 2000;40:133-41.

10 Baker R, Reddish S, Robertson N, et al. Randomised controlled trial of tailored strategies to implement guidelines for the management of patients with depression in general practice. Br J Gen Pract 2001;51:737-41.

11 Richards T, Richards L. QSR NUD*IST. Version 6. London: Sage, 1994.

12 Muhr T. ATLAS/Ti for Windows. Version 4.2. 1996.

13 Krueger RA. Focus groups. A practical guide for applied research. London: Sage Publications, 1988.

14 Donovan J, Blake D. Qualitative study of interpretations of reassurance among patients attending rheumatology clinics: "just a touch of arthritis, doctor?" BM 2000;320:541-4.

15 Kavanagh A, Broom D. Women's understanding of abnormal cervical smear test results: a qualitative interview study. BM 1997;314:138891.

16 Naish J, Brown J, Denton, B. Intercultural consultations: investigation of factors that deter non-English speaking women from attending their general practitioners for cervical screening. BM 1994;309:1126-8.

17 Grol R. Personal paper: beliefs and evidence in changing clinical practice. BM 1997;315:418-21.

18 Carthy P, Harvey I, Brawn R, et al. A study of factors associated with cost and variation in prescribing among GPs. Fam Pract 2000;17:36-41.

19 Coenen S, Van Royen P, Vermeire E, et al. Antibiotics for coughing in general practice: a qualitative decision analysis. Fam Pract 2000; 17:380-5.

20 Hughes D, Griffiths L. 'Ruling in' and 'ruling out': two approaches to the micro rationing of health care. Soc Sci Med 1997;44:589-99.

21 Plsek P. Redesigning health care with insights from the science of complex adaptive systems. In: Institute of Medicine. Crossing the quality 
chasm. A new health system for the 21 st century. Washington DC National Academy Press, 2001: 309-22.

22 Abu J, Baker R, Habiba M, et al. Qualitative and quantitative assessment of women's views of a one-stop menstrual clinic compared to traditional gynaecology clinics. Br J Obstet Gynaecol 2001:108:993-9.

23 Preston C, Cheater F, Baker R, et al. Left in limbo: patients' views on care across the primary/secondary interface. Qual Health Care 1999;8:16-21.
24 Van Wersch A, Eccles M. Involvement of consumers in development of evidence based clinical guidelines: practical experiences from the North of England evidence-based guideline development programme. Qual Health Care 2001:10:10-16.

25 Katbamna S, Baker R, Ahmad W, et al. Development of guidelines to facilitate improved support of South Asian carers by primary health care teams. Qual Health Care 2001;10:166-72.

\section{SEMANTICS}

\section{"Quality improvement": noun or verb?}

$\longrightarrow$ an a collection of activities be categorized as "quality improvement"? Is it research? Practice? Do we need to obtain informed consent from patients if we are soliciting their feedback to change our practices? It started me puzzling on the evolution of our use of language in the field.

The words we attach to ideas and experiences shape our thinking about them. ${ }^{1}$ The terms "quality improvement", "quality improvement project", "QI tools", "QI methods", a "quality improvement activity" and many other examples of using the words "quality" and "improvement" in a noun or adjectival form have helped people to name and understand what was new about some new activities and energies to change and improve health care. I wonder if the way we use these words is changing?

The words "quality" and "improvement" are not new. From the early 14th century Latin "quālis" and the later French "qualitie", our modern word "quality" began its modern life as a word that referred to "of what sort?" and, since before 1400, "grade of excellence". More recently in the 17th century the root "enprowment" of our word "improvement" began by referring to "good or profitable use". In 1647 the meaning of "betterment" is recorded and relates to a specialized meaning of improving land for better uses. ${ }^{2}$

As we used the words "quality improvement" initially in health care, they were associated with some constructs new to health care such as "organization-wide quality efforts" which implied new roles for leaders. ${ }^{3-6}$ For some there were new ways of understanding health care giving as a process and system, ${ }^{78}$ and for others there were new "tools" such as flow charts or control charts. ${ }^{910}$ Initially it seemed appropriate to me to use the words "quality improvement" to name or define-as nouns do-a new category of ideas, methods, activities, and other phenomena.

Today we are improving quality by taking actions. "Quality improvement" is no longer an abstract collection of ideas. We have some experience of translating these ideas into the real practice of health care. We act to learn about and integrate the preferences of patients in shared decisions about daily care. We act to understand and try tests of change in the care systems where patients and caregivers meet. We act to measure and learn from the study of variation in process and outcome. We act to integrate caregivers from across disciplines and with information and information technology. Some wish to classify or label these actions as "QI" for other purposes such as for external review or for the purpose of getting informed consent.

This imperative-relentlessly to study and improve quality-has been part of what it means to be a "health professional" from antiquity to recent times. ${ }^{11}{ }^{12}$ The Accreditation Council of Graduate Medical Education (ACGME) and the American Board of Medical Specialties (ABMS) in the US have restated this recently in accrediting and certifying procedures. For example, in the US it is expected that healthcare professionals be competent in "practice-based learning and improvement" and "systems-based practice" as part of their graduate medical education and certification (www.acgme.org and www.abms.org). Organizations in the US that seek certification and accreditation as healthcare organizations are expected to be engaged in the continual improvement of the care they offer (www.jcaho.org). For many, these expectations of professional action are not new, just restated in what seems like a more complex world. As we increasingly calibrate our efforts by measured outcomes, we realize the broad array of actions that may be necessary to improve the quality of health care in complex systems. So, how should we think of the words "quality improvement"?

When we use language to help give meaning and to make sense of situations, the words themselves take on new nuances to meet our needs. By creating a vocabulary for the field of "quality improvement", we need to avoid creating a world abstract and unrelated to the practical work of improving quality. We spend valuable energy debating whether or not some activity can or cannot be considered under the noun heading "quality improvement". More importantly, I suspect, is joining forces to take necessary action to improve the delivery of health services. Let us think about "quality improvement" as a verb, as action that we take to bring about better outcomes (ultimately) for patients. Let us judge our efforts as we evaluate other verbs-by their effects on our patients and our systems.

P Batalden Center for the Evaluative Clinical Sciences, Dartmouth Medical School, Hanover, USÁ

\section{References}

1 Pinker S. The language instinct. 1st ed. New York: Harper Perennial, 1995.

2 Barnhart RK, Steinmetz S. Chambers dictionary of etymology. Edinburgh: Chambers, 1999.

3 Deming WE. Out of the crisis. Cambridge, Mass: Massachusetts Institute of Technology Center for Advanced Engineering Study, 1986.

4 Juran JM. Managerial breakthrough; a new concept of the manager's job. New York: McGraw-Hill, 1964.

5 Mizuno S. Company-wide total quality control. Tokyo: Asian Productivity Organization, 1988.

6 Feigenbaum AV. Total quality control. 3rd ed. New York: McGraw-Hill, 1991.

7 Deming WE. The new economics for industry, government, education. 2nd ed. Cambridge, Mass: Massachusetts Institute of Technology Center for Advanced Engineering Study, 1994.

8 Batalden P, Mohr J. Building knowledge of health care as a system. Qual Manage Health Care 1997;5:1-12.

9 Plsek PE. Quality improvement project models. Qual Manage Health Care 1992;1:69-81.

10 Batalden PB, Stoltz PK. Performance improvement in health care organizations-a framework for the continual improvement of health care: building and applying professional improvement knowledge to test changes in daily work. J Qual Improve 1993;19:424-52.

11 Hippocrates and Adams F. The genuine works of Hippocrates. London: Sydenham Society, 1849.

12 Nightingale F. Notes on nursing: what it is, and what it is not. London: Harrison, 1860 . 